\title{
Focal conduction block in compression neuropathy of the proximal sciatic nerve
}

\author{
Akira Inaba, Tetsuo Komori, Katsuhiko Yamada, Kazuhiko Hirose, Takanori Yokota
}

Department of

Neurology, Tokyo

Metropolitan

Neurological Hospital,

Fuchu, Tokyo

A Inaba

T Komori

K Yamada

Department of

Neurology, Tokyo

Metropolitan Fuchu

Hospital, Fuchu,

Tokyo

$\mathrm{K}$ Hirose

Department of

Neurology, Tokyo

Medical and Dental

University, Bunkyo,

Tokyo

T Yokota

Correspondence to:

Dr Akira Inaba, Department

of Neurology, Tokyo

University, 1-5-45 Yushima

University, 1-5-45 Yushi

Junky.

Received 11 July 1994

and in revised form

14 November 1994

Accepted 18 November 1994

\begin{abstract}
Direct percutaneous stimulation of the proximal sciatic nerve with a high voltage electrical stimulator was given to a patient with acute sciatic compression neuropathy. Results clearly showed a focal conduction block at the sciatic notch, which could not be precisely localised and quantified by conventional electrodiagnostic methods.
\end{abstract}

(₹ Neurol Neurosurg Psychiatry 1995;58:471-473)

Keywords: focal conduction block; compression neuropathy; sciatic nerve

Sciatic neuropathies caused by external compression usually occur when persons are bedridden for a long period or sit for long
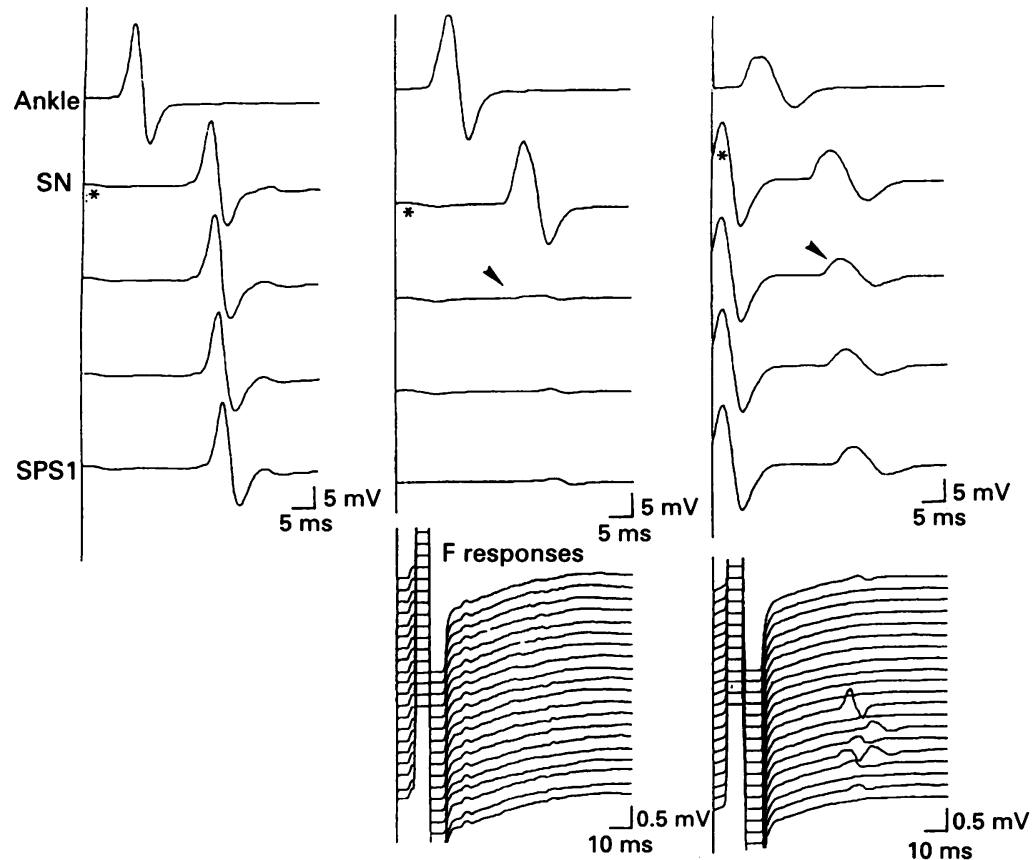

A Right tibial nerve

B Left tibial nerve

C Left peroneal nerve

Figure 1 Focal conduction block between the left sciatic notch and $6 \mathrm{~cm}$ proximal to it (arrow heads), and decreased persistence of $F$ responses in the left tibial and peroneal nerves; $C M A P$ s were recorded from the right abductor hallucis $(A)$, left abductor hallucis $(B)$, and extensor digitorum brevis (C) muscles. Upper traces; CMAPs obtained by distal stimulation at the ankle. The proximal sciatic nerve was stimulated at $6 \mathrm{~cm}$ intervals between the sciatic notch and spinous processus of S1. The early deflections at the baseline (asterisks) indicate direct responses obtained by the distal stimulation for collision. $S N=$ sciatic notch; SPS1 = spinous processus of $S 1$. periods with their buttocks pressed against a sharp edge. ${ }^{1-3}$ With conventional methods for the study of nerve conduction, it is difficult to locate the compression site on the proximal sciatic nerve because the nerve lies so deep that a long needle electrode is required to stimulate it directly. ${ }^{4}$ Moreover, needle electrode stimulation is invasive and is not acceptable for the short segment stimulation ${ }^{5}$ needed to detect focal conduction abnormalities.

We applied high voltage percutaneous electrical stimulation as a supramaximal shock (which cannot be given by a conventional electrical stimulator) to the proximal sciatic nerve, to detect the compression site of the sciatic neuropathy.

\section{Case report}

A 26 year old man experienced weakness of his left leg and mild sensory loss in the lateral calf when he awakened after sleeping eight hours with his left buttock compressed by the edge of a chair constructed of iron pipes. Neurological examination showed that he could not flex his left ankle or toes. Eversion and dorsiflexion of his left foot and toe extension were moderately impaired. Mild hypaesthesia and dysaesthesia were present in his left lateral calf and foot. The left ankle tendon reflex had disappeared but the patellar tendon reflex was preserved. All right leg functions were normal.

\section{Methods}

The first electrophysiological investigation was done 10 days after the onset of the symptoms.

Motor nerve conduction studies were performed on both legs. The compound muscle action potentials (CMAPs) were recorded on the abductor hallucis and the extensor digitorum brevis muscles from surface electrodes in the standard belly tendon arrangement. Nerve conduction between the ankle and popliteal fossa was evaluated by a conventional method. Twenty consecutive supramaximal electrical shocks were applied to the posterior tibial and deep peroneal nerves at the ankle and the persistence of the $F$ response was examined. The proximal sciatic nerve was stimulated with a high voltage electrical stimulator (the Digitimer D180, 


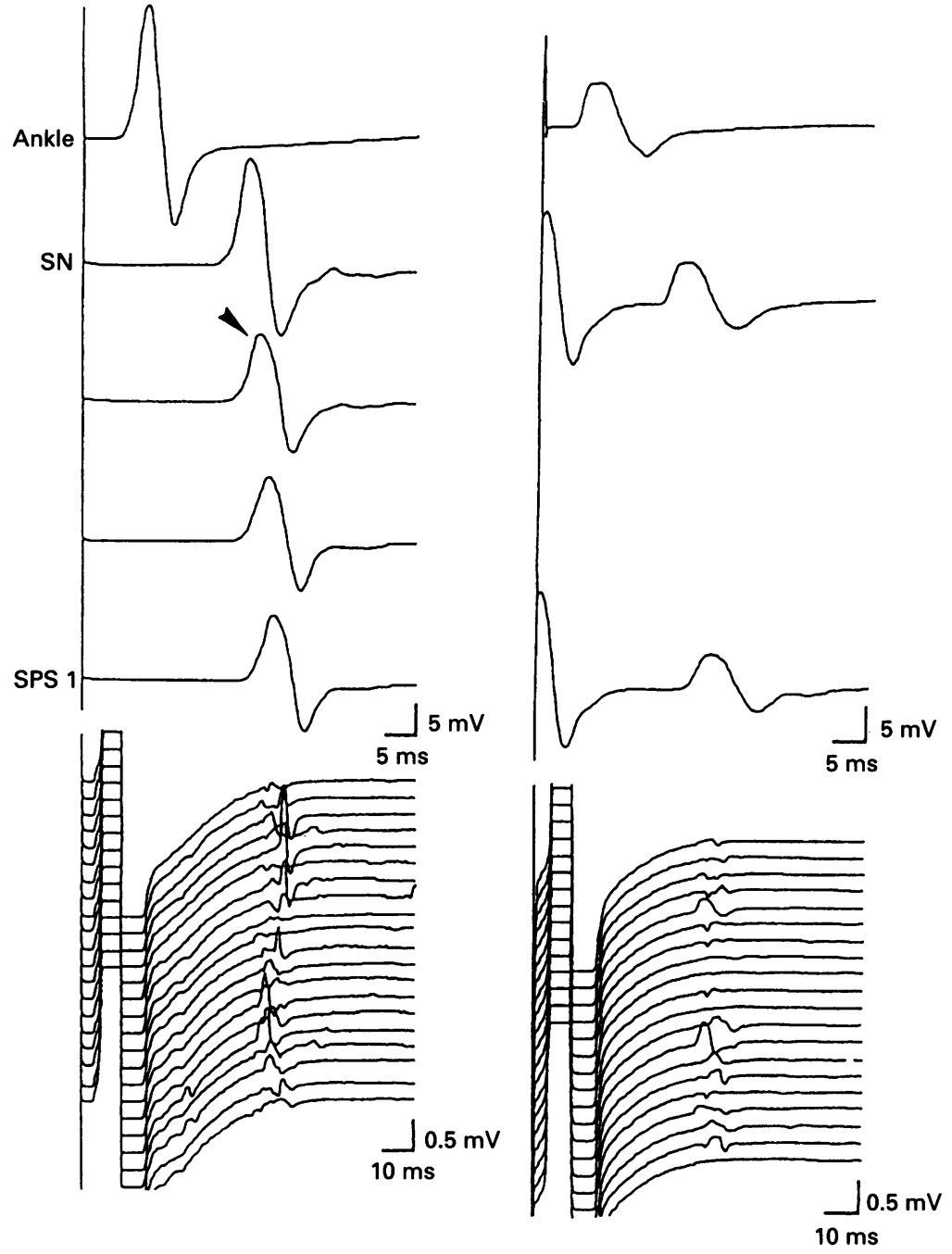

A Left tibial nerve

A Left peroneal nerve Figure 2 Nerve conduction studies done on the left tibial $(A)$ and peroneal $(B)$ nerves two months after the onset of sciatic neuropathy. The sites stimulated are the same as those in fig 1. Focal conduction block in the left tibial nerve shows lessening (arrow head). There is no conduction block in the peroneal nerve. The persistence of the F responses is improved in both nerves. firmed when there was no further increase in CMAP amplitude for a slight increase in stimulation voltage. The change of peak to peak ( $\mathrm{p}-\mathrm{p})$ amplitude and the negative peak $(-p)$ area of the CMAPs between the sciatic notch and sacral nerve root were calculated and the results compared with those for normal controls (age range 23-59 years, $n=15$ ).

In our patient, short segment stimulation was given at $6 \mathrm{~cm}$ intervals between the sciatic notch and sacral nerve root to determine the site of the conduction abnormalities.

Nerve action potentials (NAPs) of the left sural nerve were recorded antidromically. Needle electromyography (EMG) was performed in the left anterior tibial and medial head of the gastrocnemius muscles.

During the examinations, the skin temperature was maintained above $32^{\circ} \mathrm{C}$. The instrument used for the recordings and analyses was a Counterpoint (Dantec Co Ltd) electromyograph with a $30 \mathrm{~Hz}-5 \mathrm{kHz}$ frequency band.

\section{Results}

In normal controls, the $\%$ reductions in $\mathrm{p}-\mathrm{p}$ amplitude and in the $-\mathrm{p}$ area between the sciatic notch and sacral nerve root were 8.4 (SD 6.9$) \%$ and $5.6(8.3) \%$ in the tibial nerve, and $6.1(12.4) \%$ and $0.03(8.4) \%$ in the peroneal nerve. None of the controls showed more than a $30 \%$ reduction in the p-p amplitude and $-p$ area of their CMAPs in this segment.

The patient's motor nerve conductions were normal between the ankle and popliteal fossa in the tibial and peroneal nerves on both sides. Figure 1 shows results of the sciatic nerve conduction study and the $F$ responses for the patients. The persistence of the $F$ responses was greatly decreased in the left tibial nerve and moderately decreased in the left peroneal nerve. There were abnormal reductions in $p-p$ amplitude and $-p$ area between the left sciatic notch and sacral nerve root. In this segment, the respective reductions in $\mathrm{p}-\mathrm{p}$ amplitude and $-\mathrm{p}$ area were $96.8 \%$ and $94.6 \%$ in the tibial nerve, and $55.0 \%$ and $50.2 \%$ in the peroneal nerve. With short segment stimulations, we located the focal conduction block as being between the sciatic notch and $6 \mathrm{~cm}$ proximal to it (fig 1 , arrow head). There were no conduction abnormalities on the unaffected (right) side. Amplitude and conduction velocity of the NAP in the left sural nerve were normal. A needle EMG showed a severely reduced interference pattern at maximal effort with normal motor unit potentials (MUPs) in the left gastrocnemius muscle. The tibialis anterior muscles showed a mildly reduced interference pattern with normal MUPs. Spontaneous activities at rest were not found in either muscle.

Results of nerve conduction studies done two months after the onset of weakness in the patient's left leg showed that there had been considerable improvement (fig 2 and table). The $\%$ reductions of $p-p$ amplitude and $-p$ 
Percentage reduction of $p-p$ amplitude and - p area of CMAP between the sciatic notch and spinous processus of S1 of the patient and normal subjects

\begin{tabular}{llllllll}
\hline & \multicolumn{2}{l}{$\begin{array}{l}\text { First investigation } \\
\text { Amplitude }\end{array}$} & \multicolumn{2}{l}{$\begin{array}{l}\text { Second (two months later) } \\
\text { Amplitude }\end{array}$} & Area & \multicolumn{2}{l}{ Normal subjects $(n=15)$} \\
& & Amplitude & Area & \\
\hline Left tibial nerve & $96 \cdot 8$ & $94 \cdot 6$ & $33 \cdot 2$ & $33 \cdot 3$ & $8 \cdot 4(6 \cdot 9)(0 \cdot 8-28 \cdot 2)$ & $5 \cdot 6(8 \cdot 3)(0-29 \cdot 8)$ \\
Left peroneal nerve & $55 \cdot 0$ & $50 \cdot 2$ & $18 \cdot 6$ & $20 \cdot 2$ & $6 \cdot 1(12 \cdot 4)(0-18 \cdot 3)$ & $0 \cdot 03(8 \cdot 4)(0-16 \cdot 6)$ \\
\hline
\end{tabular}

Values for normal subjects are means (SD) (range).

area of his CMAPs at the site of the conduction block had improved to $33.2 \%$ and $33.3 \%$, and there was no conduction block in the left peroneal nerve. The persistence of the $F$ response had also improved in both nerves.

Percutaneous high voltage stimulation produced only moderate discomfort and was well tolerated by the patient. No adverse side effects were seen.

\section{Discussion}

Abnormal motor and sensory conduction below the knee $^{7}$ and abnormal $\mathrm{F}$ responses ${ }^{78}$ have been reported in sciatic neuropathy. For evaluating compression neuropathy, however, it is important to detect focal conduction abnormalities such as conduction block or conduction delay in the affected segment.

The percutaneous high voltage electrical stimulator developed by Merton and Morton ${ }^{9}$ can stimulate deeply lying nerve structures. ${ }^{10}$ We have shown that it is capable of stimulating the sciatic nerve between the knee and the sciatic notch. ${ }^{11}$ We have also used it to show the presence of a focal conduction block between the sciatic notch and spinal nerve root.

The normal controls showed up to $30 \%$ reduction of $p-p$ amplitude and $-p$ area of CMAP between the sciatic notch and the spinal nerve root of $S 1$. Although it is difficult to exclude the possibility that the supramaximal stimulation could not be achieved over the spinous processus of $S 1$, we think that the reduction was caused mainly by temporal dispersion and interphase cancellation of motor action potentials. The advantages of our method are its safety and non-invasiveness for short segment supramaximal stimulations. These advantages allowed repeated examinations to confirm the reproducibility of the site of conduction block and improvement in the block. Generally, greater vulnerability of the peroneal division occurs in sciatic neuropathy. ${ }^{12}$ In our patient, however, the clinical symptoms and electrophysiological investigation showed that the tibial division was much more affected than the peroneal division. Although the reason why the tibial division was affected severely in our patient is uncertain, the tibial nerve was reported to be affected dominantly in $10 \%$ of the patients. ${ }^{13}$

We think that this is the first report of direct electrodiagnostic evidence of focal conduction block in sciatic nerve compression neuropathy. Percutaneous high voltage stimulation should be of great assistance in evaluating the localisation of lesions of the sciatic nerve.

1 Stewart JD. Mononeuropathies of the lower extremities. In: Brown WF, Bolton CF, eds. Clinical electromyograIn: Brown WF, Bolton CF, eds. Clinical electromyogra1993:305-22.

2 Stewart JD, Angus E, Gendron D. Sciatic neuropathies. $B M \mathcal{F}$ 1983;287:1108-9.

3 Vogel CM, Albin R, Albers JW. Lotus footdrop: sciatic neuropathy in the thigh. Neurology 1991;41:605-6.

4 Yap CB, Hirota T. Sciatic nerve conduction velocity study. F Neurol Neurosurg Psychiatry 1967;30:233-9.

5 Cornblath DR, Sumner AJ, Daube J, et al. Conduction block in clinical practice. Muscle Nerve 1991;14:869-71.

6 Kimura J. Collision technique. Physiological block of nerve impulses in studies of motor nerve conduction velocity. Neurology 1976;26:680-2.

7 Venna N, Bielawski M, Spatz EM. Sciatic nerve entrap-

ment in a child. $\mathcal{F}$ Neurosurg $1991 ; 75: 652-4$.
8 Jones HR. Compressive neuropathy in childhood: a report of 14 cases. Muscle Nerve 1986;9:720-3.

9 Merton PA, Morton HB. Stimulation of the cerebral cortex in the intact human brain. Nature 1980;285:227.

10 Maertens de Noordhout A, Rothwell JC, Thompson PD, Day BL, Marsden CD. Percutaneous electrical stimulation of lumbosacral roots in man. $\mathcal{F}$ Neurol Neurosurg Psychiatry 1988;51:174-81.

11 Inaba A, Yokota T, Miyatake T, Komori T, Tanabe H. The sciatic nerve conduction study by percutaneous high voltage electrical stimulation. Electroencephalogr Clin Neurophysiol 1993;87:S88.

12 Katirgi B, Wilbourn AJ. High sciatic lesions mimicking peroneal neuropathy at the fibular head. $f$ Neurol $S_{c i}$ 1991;121:172-5.

13 Johnston W, Stewart JD. Sciatic neuropathy: a 10 year experience. Can F Neurol Sci 1990;17:249. 